\title{
Comparison of naturally aging and D-galactose induced aging model in beagle dogs
}

\author{
MUSI JI ${ }^{12^{*}}$, XIAOHUA SU ${ }^{1 *}$, JIZHEN LIU $^{1 *}$, YI ZHAO ${ }^{1}$, ZHIDONG LI $^{1}$, \\ XUN XU ${ }^{1}$, HUAWEN LI $^{1}$ and BAYAER NASHUN ${ }^{1}$ \\ ${ }^{1}$ Laboratory Animal Center, Guangdong Medical University, Dongguan, Guangdong 523808; \\ ${ }^{2}$ Department of Internal Medicine, Liaobu Hospital, Guangdong Medical \\ University, Dongguan, Guangdong 523400, P.R. China
}

Received May 11, 2017; Accepted October 17, 2017

DOI: $10.3892 /$ etm.2017.5327

\begin{abstract}
Animal models have been used to study aging for decades. In numerous aging studies, beagles are the most commonly used breed of dog. However, few studies have compared between naturally aging models and experimentally induced aging models in beagle dogs. In the present study, a D-galactose induced aging model was compared with a naturally aging model, and young adult dogs were considered as the young control group. The level of malondialdehyde (MDA), superoxide dismutase (SOD) and glutathione peroxidase (GSH-Px) in serum, and brain tissue were measured. Histopathological comparisons of the liver, kidneys, heart, lungs and spleen were evaluated using hematoxylin and eosin (H\&E) staining, in addition, the brain was evaluated by H\&E staining, and Nissl staining. The expression levels of aging-associated factors in the hippocampus, including proliferating cell nuclear antigen (PCNA), P16 and P21 were also determined through reverse transcription quantitative-polymerase chain reaction, and western blot analysis. The results indicated that D-galactose induced aging significantly increased the MDA level, while the levels of SOD and GSH-PX were diminished when compared with the young control group, which was similar to the naturally aging group. Parallel histopathological features were observed in the D-galactose induced aging and naturally aging groups compared with the young control group. However, a reduced expression level of PCNA, and increased expression levels of P16 and P21 were observed in the naturally ageing and induced aging groups compared with the young control group. The results of the
\end{abstract}

Correspondence to: Dr Bayaer Nashun, Laboratory Animal Center, Guangdong Medical University, 1 Xincheng Road, Songshan Lake Hi-tech Industrial Development Zone, Dongguan, Guangdong 523808, P.R. China

E-mail: bayaer17@yeah.net; nashunbayaer@163.com

*Contributed equally

Key words: aging, animal model, beagle dogs, induced aging current study demonstrated that the beagle dogs in D-galactose induced aging model exhibited significant similarities with the naturally aging model, providing evidence to support that the D-galactose induced aging model may be applied to aging studies.

\section{Introduction}

Aging is associated with a progressive decline in a number of physiopathological processes, leading to health complication and disease, including cardiovascular diseases, cancer and neurodegenerative diseases (1). With the continuous increasing global aging population, aging has already become a huge society burden. How to reverse aging has become one of the biggest challenges in clinical and biological research. In order to understand the basic mechanism of aging as well as the physiopathological and behavioral effects of aging, aging studies range from the molecular level to the global organism level, from cell to the whole body, which are extensively investigated worldwide (2,3). Animal aging models are important for aging study, and several standard models have been established, including fruit fly, fish, birds, mouse, rats and dogs (4,5). Generally, aging models can be classified into two categories: naturally aging model and accelerated aging models $(6,7)$. Due to practical condition of the research projects, such as duration and budget, accelerated aging models are widely and commonly used $(6,8)$.

Among mammal aging models, beagle dogs have been generally used. They have moderate life spans, varying from approximately $12-15$ years, and they are easy to handle and present in abundance (9). Aged beagle dogs are an excellent model in many respects, since dogs and human share a number of chronic age-associated ailments, including mobility decline, cardiac dysfunction, neurodegenerative disorders and other chronic organ failure (10-12). Despite, as an aging model, the duration of aging studies using beagle dogs is up to 6-8 years, which is time consuming and leads to a huge expenses. Therefore, establishment of accelerated aging models is important for improving the efficiency of aging studies using beagle dogs. Experimental-induced aging by chemical compounds, such as D-galactose, has been used as accelerated aging models in mouse and rats $(13,14)$. D-galactose is a 
reducing sugar and an important nutrient that react with amino acids to form glycation end products through non-enzymatic glycation. D-galatcose also contributes to generation of reactive oxygen species (ROS) via metabolism of D-galactose (15). It has been showed that D-galactose induced aging mice is observed progressive declines in learning and memory ability, mobility and cardiac dysfunction, which is similar to naturally aging mice (16). However, there are very few studies using D-galactose induced aging in beagle dogs.

In the present study, we induced aging in young adult beagle dogs using D-galactose. This D-galactose induced aging model was compared with naturally aging beagle dogs, and young adult beagle dog was used as control. Our data revealed that D-galactose induced aging group had an increased MDA level and decreased SOD and GSH-Px level, which is similar to the naturally aging group in comparison to young control group. Parallel histopathological features were observed in both D-galactose induced aging and naturally aging group compared with young control group. Reduced expression level of proliferating cell nuclear antigen (PCNA), and increased expression levels of P16 and P21 in the hippocampus were observed in both naturally ageing group and induced aging group compared with the young control group. Our observations provides a strong piece of evidence supporting that D-galactose induced aging model as a well-established model in mice and rats, can also be applied in beagle dogs. In addition, our comparison study extends the understanding of the etiology of aging-related progression in different organs and also the important implication in the investigations on age-related diseases.

\section{Materials and methods}

Animals and treatment. Beagle dogs of both genders were provided by Guangzhou General Pharmaceutical Research Institute,Ltd.(Guangzhou,China) and housed in the Guangdong Medical University Animal Center (Zhanjiang, China). Total 14 dogs were used in this study, including 4 dogs at 10 years of age ( 2 male and 2 female) as naturally aging group, 6 dogs at 2 years of age ( 3 male and 3 female) as induced aging group and 4 dogs at 2 years of age ( 2 male and 2 female) as young control group. They were kept under controlled conditions (room temperature, $22-24^{\circ} \mathrm{C}$; relative humidity, $40-60 \%$ ) and accustomed to the housing environment for 1 week prior to the experiment with free access to food and distilled water. The induced aging group was given D-galactose at $50 \mathrm{mg} / \mathrm{kg}$ daily subcutaneous injections for 90 days. Blood samples from each group were collected every four weeks. Animals were sacrificed on the last day of treatment, and blood, sera and organs were immediately collected for bioassay or stored at $-70^{\circ} \mathrm{C}$ for later use. All the experimental procedures were conducted following the approval of the Animal Ethics Committee of Guangdong Medical University.

MDA content, SOD activity and GSH-Pxactivity measurement. serum sample and homogenates from brain tissue were assayed for the determination of oxidative stress-related factors including malondialdehyde (MDA) and superoxide dismutase (SOD) and glutathione peroxidase (GSH-Px) by kits (Nanjing Jiancheng Bioengineering Institute, Nanjing, China) using an automatic chemical analyzer according to manufacturer's instructions.

Hematoxylin and eosin $(H \& E)$ staining. A segment of tissue (liver, kidney, heart, lung and spleen and brain) was transferred to the fixative (4\% formaldehyde and $1 \%$ glutaraldehyde in PBS, pH 7.4). The fixed segment of tissue was embedded in paraffin, $5 \mu \mathrm{m}$ thick sections were cut by Sliding microtome Leica SM2010 R (Leica Store Shanghai, Shanghai, China). Sections were dehydrated by gradient ethanol and stained with $\mathrm{H} \& \mathrm{E}$. Images were captured by microscope.

Nissl staining. Hippocampus tissue was removed from the brain and fixed with $4 \%$ paraformaldehyde for $48 \mathrm{~h}$. Sections (10- $\mu$ m thick) were cut on a cryostat (Leica Microsystems $\mathrm{GmbH}$, Wetzlar, Germany). The sections were hydrated in $1 \%$ toluidine blue at $50^{\circ} \mathrm{C}$ for $20 \mathrm{~min}$ for Nissl staining. Then they were dehydrated and mounted with Permount (Thermo Fisher Scientific, Inc., Waltham, MA, USA) after rinsing with distilled water. The slide was observed under a microscope.

Immunohistochemical staining. Immunostaining of iNOS, COX-2 and NF- $\mathrm{NB}$ was carried out on the tissue from liver, kidney, heart, lung and spleen and brain with PBS containing 0.05 M EDTA followed by $4 \%$ paraformaldehyde. Then $5-\mu \mathrm{m}$ sections were incubated with blocking reagent followed by primary antibody anti-iNOS or anti-COX-2 or anti-NF- $\mathrm{BB}(17)$ in the presence of $10 \%$ rabbit serum overnight at $4^{\circ} \mathrm{C}$, followed by biotin-conjugated goat anti-rabbit Ig, avidin-linked HRP complex and 3,3'-diaminobenzidine as substrate. Slides were counterstained with hematoxylin, dehydrated, and examined under a light microscope. NIH ImageJ was used for macrophage and neutrophil quantification.

Western blot analysis. Tissue samples from the brain were harvested and stored at $-80^{\circ} \mathrm{C}$. For western blot analysis, frozen samples were sonicated on ice twice for $5 \mathrm{sec}$ in $50 \mathrm{mM}$ lysis buffer (pH 7.4) containing $3.1 \mathrm{mM}$ sucrose, $1 \mathrm{mM}$ DTT, $10 \mu \mathrm{g} / \mathrm{ml}$ leupeptin, $10 \mu \mathrm{g} / \mathrm{ml}$ soybean trypsin inhibitor, $2 \mu \mathrm{g} / \mathrm{ml}$ aprotinin, and $0.1 \%$ Triton X-100. Homogenates were centrifuged at $10,000 \mathrm{x}$ g at $4^{\circ} \mathrm{C}$ for $20 \mathrm{~min}$ and the supernatant was collected. The total protein concentration was measured using the Bradford protein assay (Bio-Rad Laboratories, Inc., Hercules, CA, USA). Protein lysates $(20 \mu \mathrm{g})$ was separated using $12 \%$ SDS-PAGE and transferred to a PVDF membrane. After blocking with 5\% nonfat milk, the PVDF membrane was incubated overnight with the primary antibody as follows: rabbit anti-PCNA, rabbit anti-p21 and rabbit anti-p16 solute in TBS-T. Membranes were washed in TBS-T (10 min x3) and then probed with the appropriate secondary antibody (1:10,000; Abcam, Cambridge, UK). Membranes were developed using Versa Doc 5000 and band densities were measured with Quantity One 4.6 software (Bio-Rad Laboratories, Inc.). Equal protein loading was additionally verified by measurement of GAPDH level with rabbit polyclonal antibody.

Gene expression analysis using qPCR. The total RNA from tissue samples was extracted by using TRIzol kit (Invitrogen; Thermo Fisher Scientific, Inc.) according to the manufacture instruction. A total of $1 \mu \mathrm{g}$ RNA was reverse transcribed 

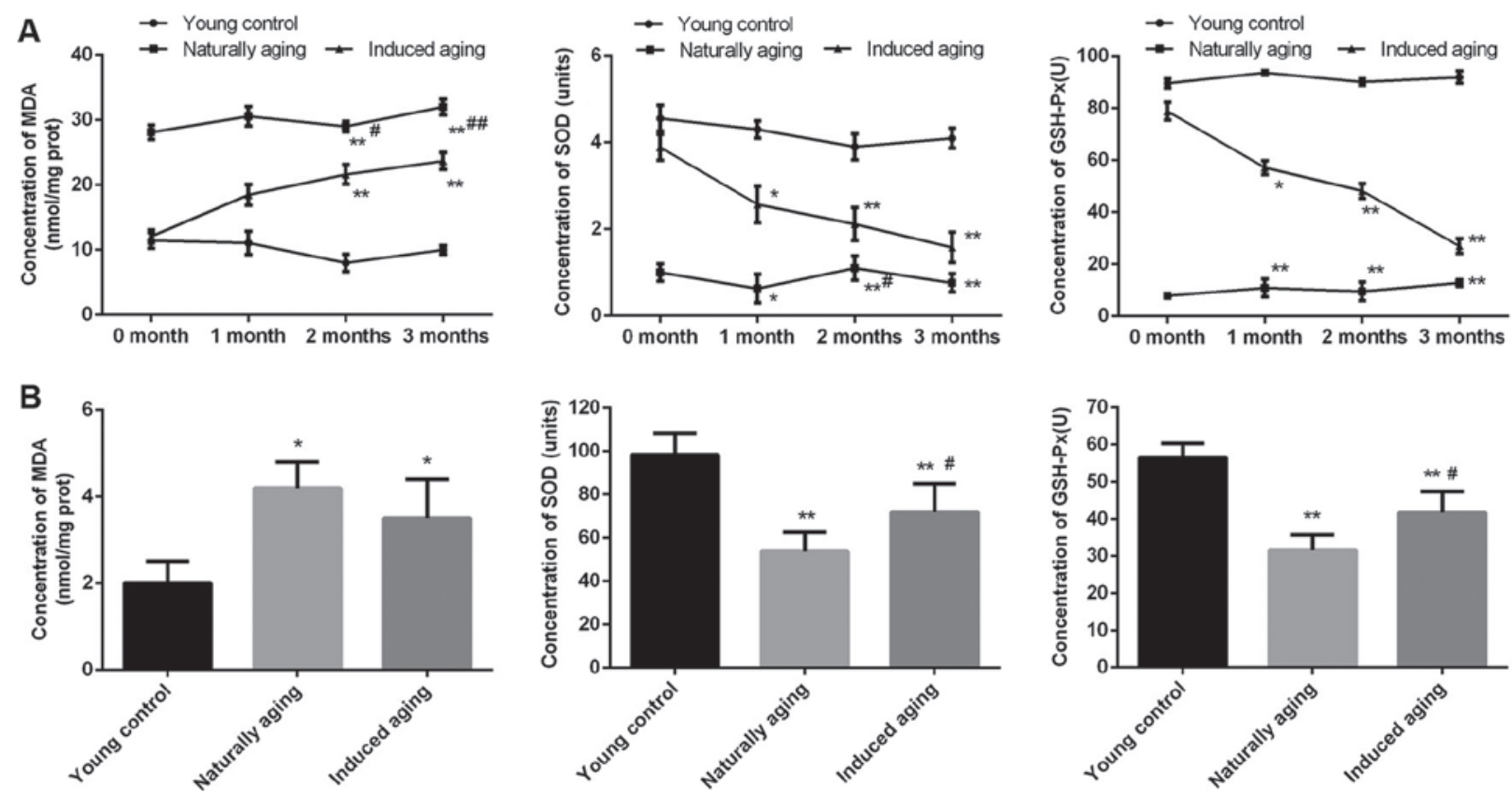

Figure 1. Concentration of MDA, SOD and GSH-Px in the induced aging group and naturally aging group compared to the young control group. (A) The levels of MDA, SOD and GSH-Px in the serum. (B) The levels of MDA, SOD and GSH-Px in the brain. " $\mathrm{P}<0.05$ and ${ }^{* * *} \mathrm{P}<0.01$ vs. young control group; ${ }^{\mathrm{P}} \mathrm{P}<0.05$ and ${ }^{\# \#} \mathrm{P}<0.01$ naturally aging vs. induced aging group. MDA, malondialdehyde; SOD, superoxide dismutase; GSH-Px, glutathione peroxidase.

into complementary (c)DNA using oligo (dT) and III reverse transcriptase is a version of M-MLV RT. Real-time PCR was carried out with an ABI 7500 Real-Time PCR system. Target cDNA levels were determined by SYBR Green-based real-time PCR in $20 \mu 1$ reactions containing $10 \mu 1$ Power SYBR Green Master Mix (Applied Biosystems; Thermo Fisher Scientific, Inc.). PCNA forward, 5'-TGTCGATAAGGAGGAGGA AGC-3' and reverse, 5'-CTGTAGGAGAGAGCGGAGTGG-3'; p16 forward, 5'-ACCCCGTCACCCTCACC-3' and reverse, 5'-GCTCCTCAGCCAGGTCCAC-3'; p21 forward, 5'-GGG GACAGCTCAGGAGGA-3' and reverse, 5'-CGGCGTTTG GAGTGATAGA-3' and GAPDH forward, 5'-GGTCGGAGT GAACGGATTT-3' and reverse, 5'-CATTTGATGTTGGCG GGA-3'. The general PCR cycle was performed as follows: $2 \mathrm{~min}$ at $94^{\circ} \mathrm{C}, 29$ to 32 cycles of $95^{\circ} \mathrm{C}$ for $30 \mathrm{sec}, 57-60^{\circ} \mathrm{C}$ for $45 \mathrm{sec}, 72^{\circ} \mathrm{C}$ for $1 \mathrm{~min}, 72^{\circ} \mathrm{C}$ for $5 \mathrm{~min}$ and hold at $4^{\circ} \mathrm{C}$. The qPCR data were analyzed with a $2^{-\Delta \Delta C t}$ method and normalized using $G A P D H$ as an internal control.

Statistical analysis. Statistical calculations were performed using Prism 6 (GraphPad Software, Inc., La Jolla, CA, USA). Data are presented as the mean \pm SEM. Student's t-tests were used for comparisons between 2 groups, and one-way or two-way ANOVA was used for comparisons among multiple groups. $\mathrm{P}<0.05$ or $\mathrm{P}<0.01$ were considered to indicate a statistically significant result.

\section{Results}

Comparison of oxidative stress markers. In order to evaluate the level of oxidative stress in the induced aging group and naturally aging group, MDA content, SOD activity and GSH-Px activity as oxidative stress markers were determined. In the induced aging group, MDA level was progressively increased from about $60 \%$ after one month D-galactose treatment to almost $120 \%$ after three months treatment in comparison to young control group. While SOD activity and GSH-Px activity were gradually decreased by approximately 100 and $75 \%$ after three months treatment compared with young control group, representatively. In the naturally aging group, steady high level of MDA and steady low level of SOD activity and GSH-Px activity were observed, compared with young control group (Fig. 1A). Similar results were obtained from the brain tissue sample. Significantly increased MDA level was detected, along with remarkably reduced SOD activity and GSH-Px activity in comparison to young control group (Fig. 1B).

Histologic expressions and histopathological features in the induced aging model. To determine histologic expressions and histopathological features in the induced aging model, H\&E staining was used for tissue morphology in liver, kidney, heart, lung and spleen and brain tissue, and nissl staining was used for morphology and pathology of neural tissue, and immunohistochemical staining was used to evaluate the expression of PCNA, p16 and p21. H\&E staining and nissl staining revealed that induced aging group and naturally aging group have parallel histopathological features in liver, kidney, heart, lung and spleen and brain: i) in H\&E stained section from lung tissue, lung injury such as inflammatory cell infiltration and alveolar wall destruction was observed in both induced aging group and naturally aging group, compared to the normal lung architecture in young control group (Fig. 2A); ii) in H\&E stained section from liver tissue, microabscess involving a few hepatocytes with inflammatory cells and necrotic debris was accumulated in the liver tissue in induced aging group and naturally aging group (Fig. 2B); iii) in $\mathrm{H} \& \mathrm{E}$ stained section from spleen tissue, we observed high degree of morphologic 

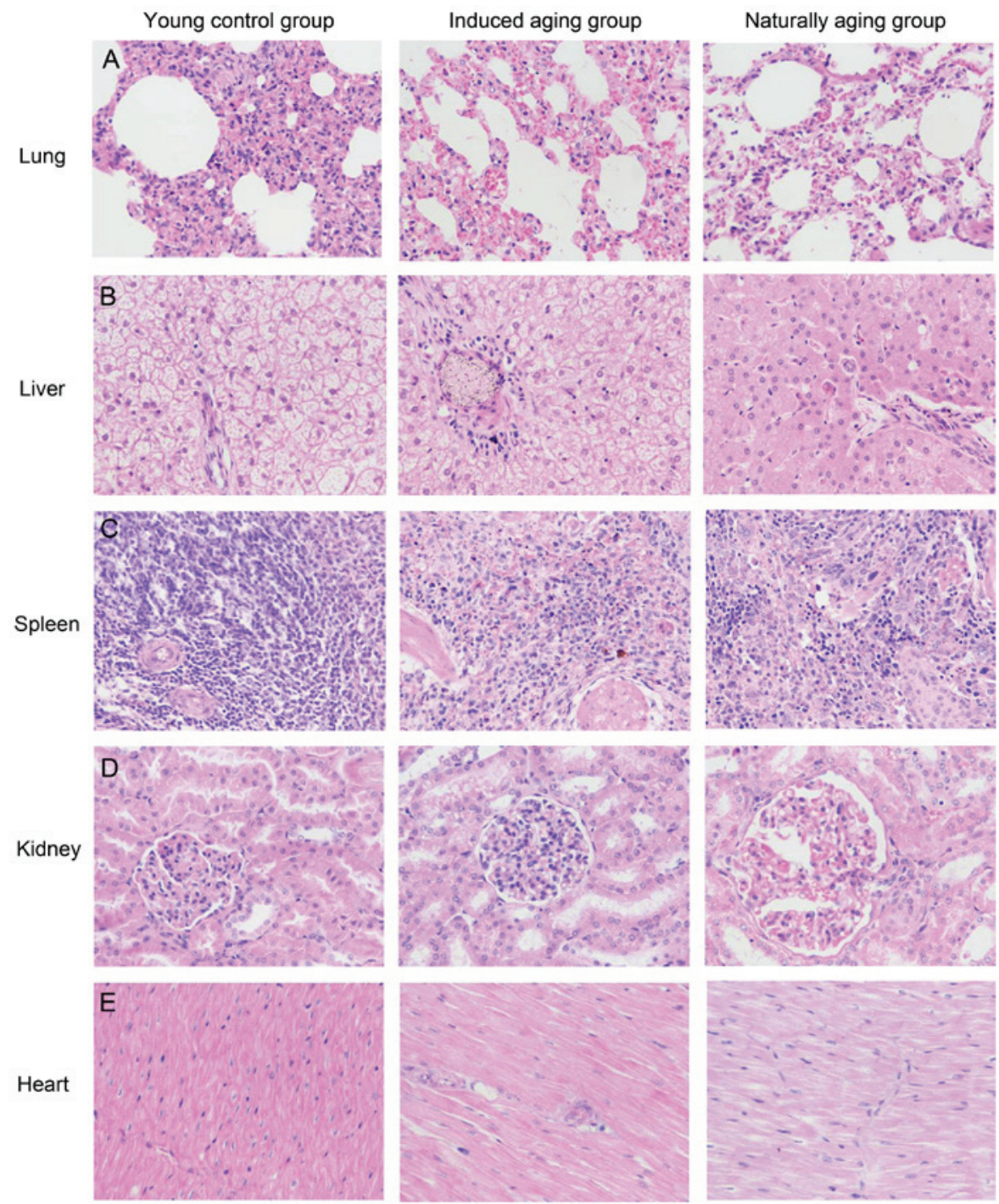

Figure 2. Histopathologic examinations on (A) lung, (B) liver, (C) spleen, (D) kidney and (E) heart at the end of the experiments (H\&E staining, $\mathrm{x} 40$ ). H\&E, hematoxylin and eosin.

A
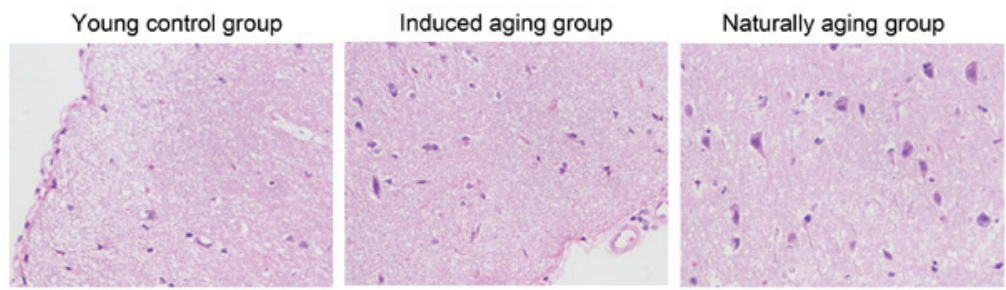

B
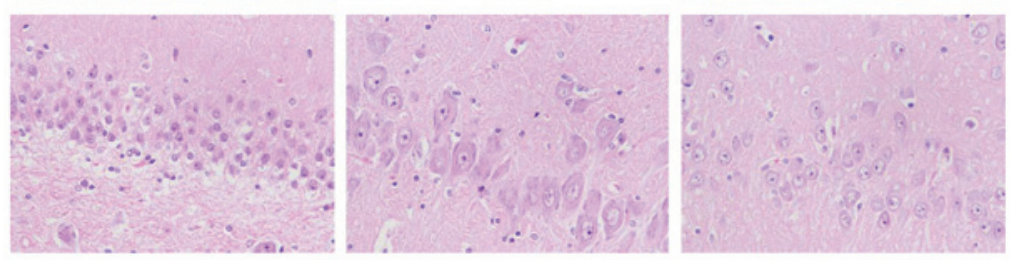

C
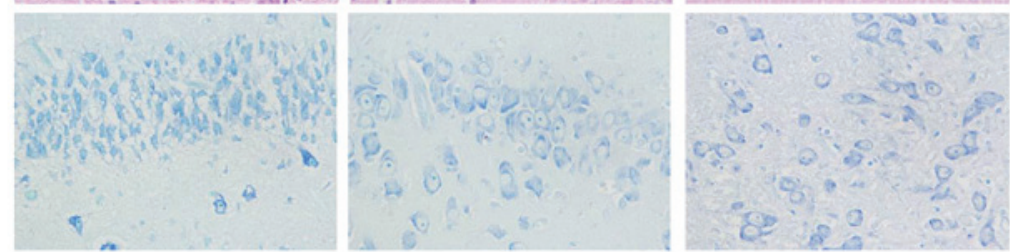

Figure 3. Histopathologic examinations on (A) cerebral cortex of hippocampus and (B and C) CA sector of hippocampus (H\&E staining, $x 40$, Nissl staining, $\mathrm{x} 40)$. H\&E, hematoxylin and eosin. 


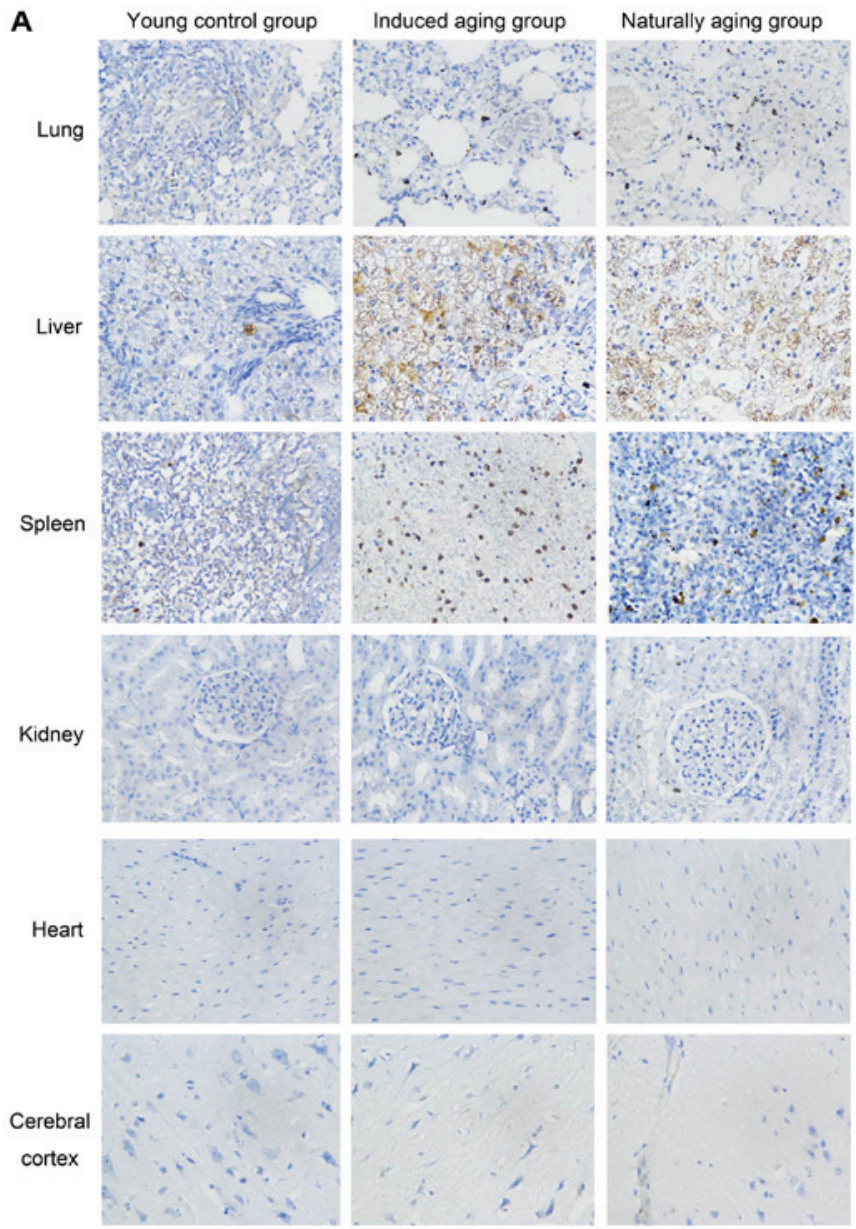

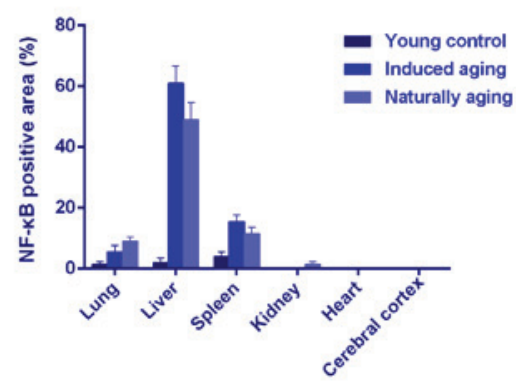

Figure 4. (A) Immunohistochemical staining of NF-kB on lung, liver, spleen, kidney, heart and cerebral cortex (x40). (B) Quantification of NF-kB-positive staining.

organization in the young control group, while density of inflammatory cells was diminished in both induced aging group and naturally aging group (Fig. 2C); iv) in H\&E stained section from kidney tissue, young control group exhibited normal histology, and induced aging and naturally aging group revealed enlarged vascular glomeruli, widening the glomerular capsular space and destructed flat epithelium lining of Bowman's capsule (Fig. 2D); v) in H\&E stained section from heart tissue, disordered arrangement and enlarged size of cardiomyocytes was observed, indicating a cardiac remodeling event in both aging groups (Fig. 2E); and vi) in sections from brain tissue, H\&E stained cerebral cortex sections from both aging groups revealed high level of inflammatory cell in comparison to young control group, which indicate that inflammation was progressing in the cerebral cortex. In H\&E

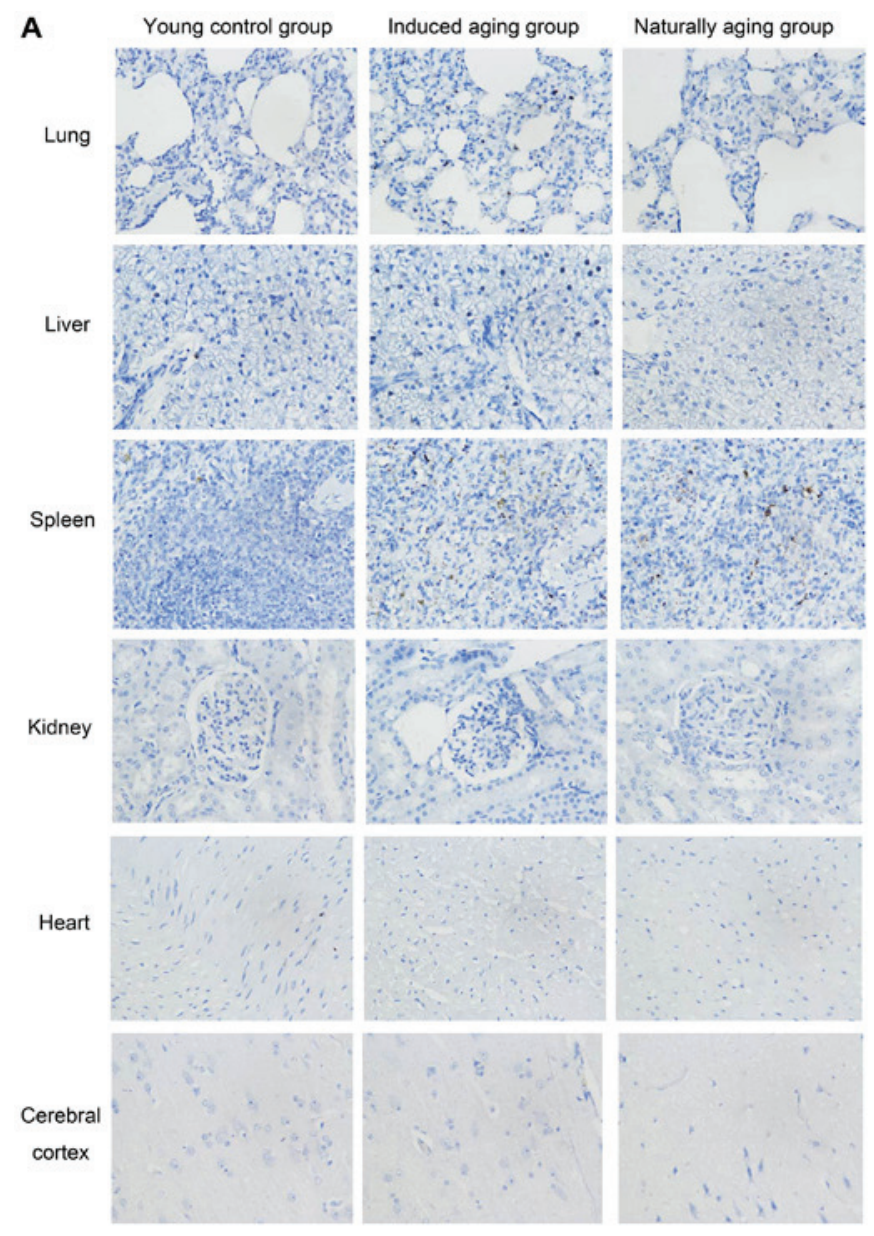

B

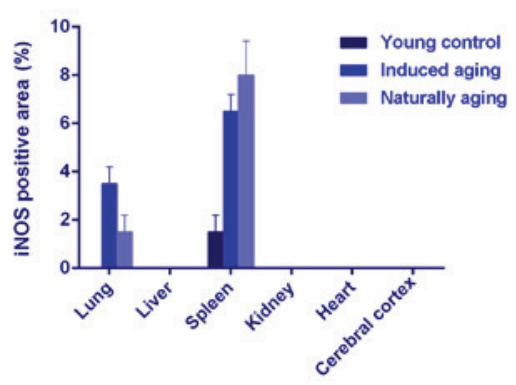

Figure 5. (A) Immunohistochemical staining of iNOS on lung, liver, spleen, kidney, heart and cerebral cortex (x40). (B) Quantification of iNOS-positive staining.

stained section of hippocampus AC area, young control group showed well organized pyramidal cells, and different types of the neuralgia cells are scattered inside the neuropil matrix, while both aging groups showed apparent decrease in the number of pyramidal cells with presence of some shrunken degenerated cells. Similar results were also observed in nissl staining sections (Fig. 3). Immunohistochemical staining indicated increased levels of NF-Kb, iNOS and COX2 in lung, liver and spleen of both aging groups in comparison to young control group (Figs. 4-6). $\mathrm{P}<0.05$, significantly different from the control groups.

Comparison of the expression of age-associated factors. The expression level of PCNA, p16 and p21 were determined by western blot and RT-qPCR. Western blot analysis indicated 


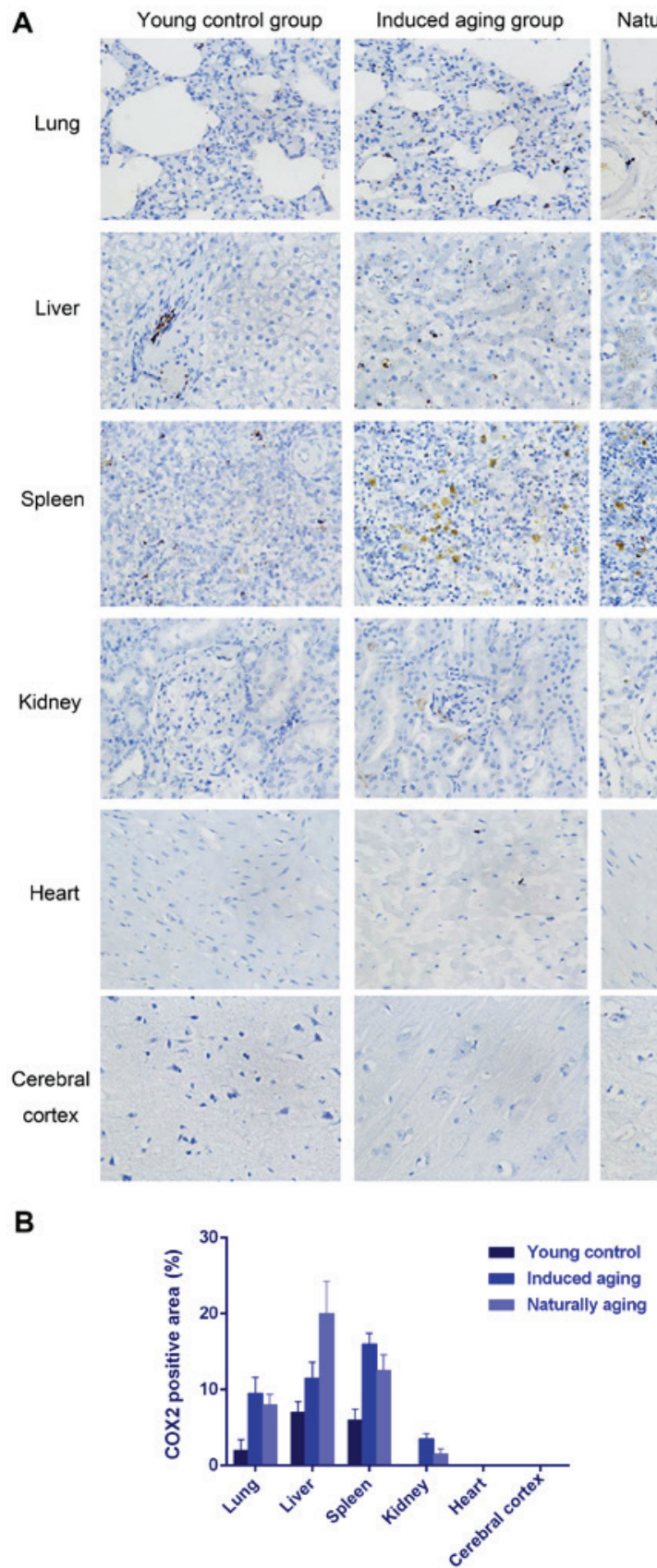

Figure 6. (A) Immunohistochemical staining of COX2 on lung, liver, spleen, kidney, heart and cerebral cortex (x40). (B) Quantification of COX2-positive staining.

that the expression level of PCNA was significantly reduced in induced aging group and naturally aging group compared with the young control group. In contrast, lower levels of p16 and p21 were detected in both aging groups in comparison to young control group. Similar results were also obtained by qPCR (Fig. 7). P<0.05, significantly different from the control groups.

\section{Discussion}

With the growing aging population, the burden of aging and aging-associated diseases will continue to be huge medical and financial problems for the society. Aging-associated pathological conditions are critical risk factors for a variety of diseases, including cancers, neurodegenerative diseases, chronic organs failure, metabolic diseases and Loss of mobility $(18,19)$. Determining the fundamental underlying cellular and molecular mechanisms of aging progression is crucial for the development of anti-aging strategies if we are to meet the increasing healthcare needs of the growing aging population. Aging models are commonly used in aging studies, since the duration of the study is a major difficulty in human aging. In order to establish a robust induced aging model in beagle dogs, in the present study, D-galactose was used to induce aging in beagle dogs as a induced aging model, and we compared histologic expressions, histopathological features and age-related factors expression with naturally aging beagle dogs model. This is the first study on comparison of naturally aging and D-galactose induced aging in beagles. Our results showed that induced aging model and naturally aging model 

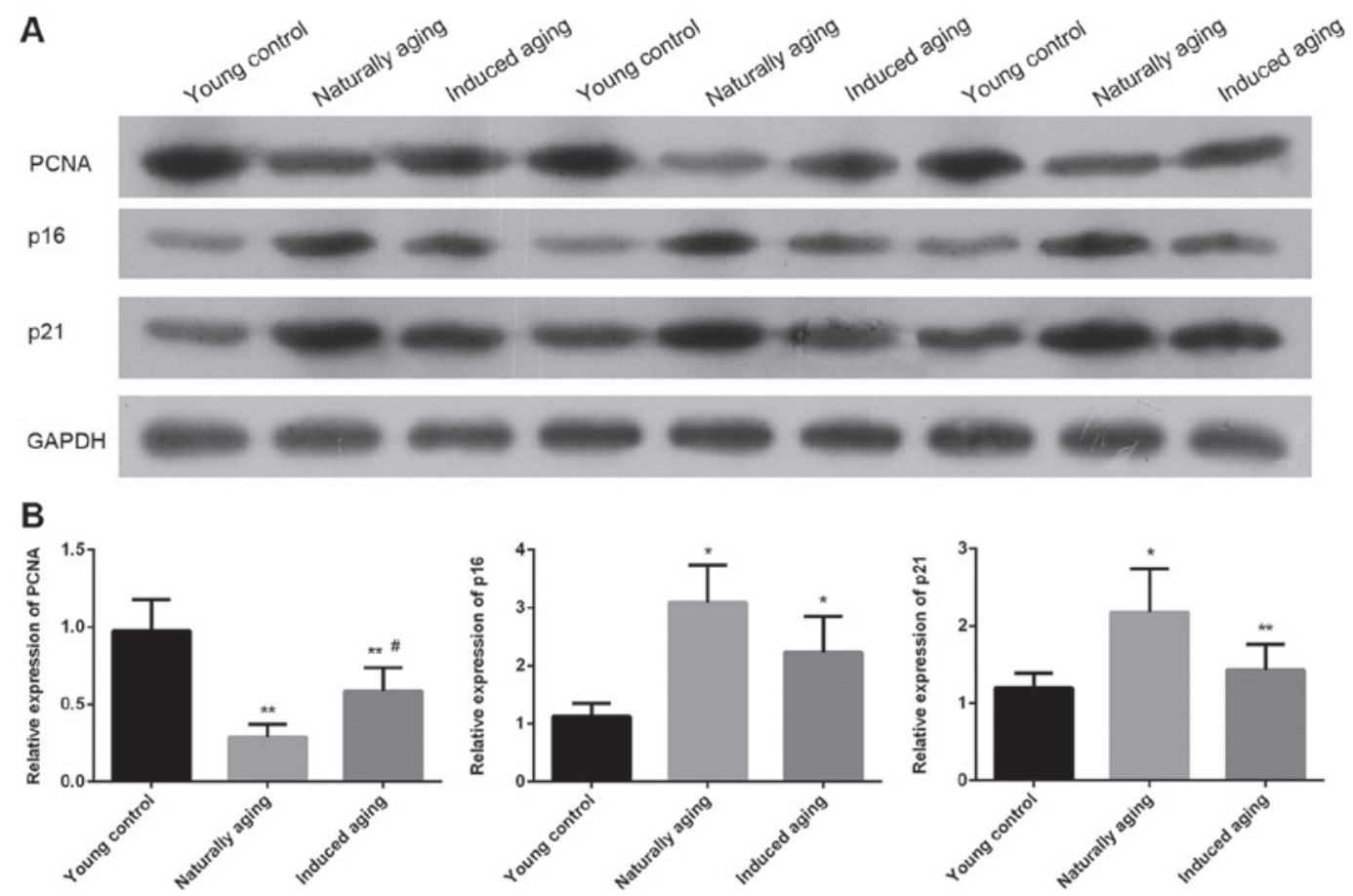

Figure 7. Changes in the expression level of PCNA, p16 and p21. (A) Western blot analysis of the level of PCNA, p16 and p21. Equal protein loading was verified by measurement of GAPDH level. (B) The relative expression level of PCNA, p16 and p21 were indicated by qPCR. " $\mathrm{P}<0.05$, ${ }^{* *} \mathrm{P}<0.01$ vs. young control group, ${ }^{\text {"}} \mathrm{P}<0.05$ vs. naturally aging group. PCNA, proliferating cell nuclear antigen.

exhibited parallel morphology and histology as well as similar level of oxidative stress related factors (MDA, SOD, GSH-Px and iNOS), age-related factors (PCNA, p16, p21 and COX2), providing a piece of evidence to support that D-galactose induced aging beagle dogs can be used for aging research in order to shorten the duration of study.

Animal aging models have been at the forefront of aging research, and obtained a wealth of information, supporting investigators to discover pathways that drive human aging (4). Successful animal aging model include mice, rats, zebrafish and dogs (5). In the present study, we used beagle dogs as model animal. Dogs as a human-company pet are ideal model for aging study, since they share the same living environment and similar food recourse. The aged dogs naturally develop age-related decline in a variety of tissue and organs and exhibit human-like individual variability in aging progression, such as muscular and neurological decline, as well as cardiovascular disease (20-23). Rodent, howerver, do not develop neurodegeneration with age (24). Therefore, dogs might be particularly interesting in the study of neurodegenerative diseases in aging (25). On the other hand, though dogs have human-like aging phenotype that makes them an attractive model, the average 10 years of life span is still problematic. Therefore, accelerated aging model or induced aging models in dogs are necessary to develop for aging studies. Accelerated aging model has been well established in mice and rats (6), and these models can be experimental induced by compounds such as dihydrotachysterol (26), O3 (27) and D-galactose (28). $\mathrm{D}$-galactose contributes to generation of reactive oxygen species (ROS) via metabolism of D-galactose. Murata et al have reported that degeneration in the retinal capillaries of galactose-fed beagle dogs results from apoptosis (29). However, there has not been a study investigating D-galactose induced accelerated aging in beagle dogs. Therefore, we treated beagle dogs with D-galactose to develop an induced accelerated aging model.

Aging-related decline or aging-associated pathological conditions contain many aspects including oxidative stress damage, chronic low-level inflammation, destructed cells and tissue lining and dysfunction of organs. Studies have showed that a significant increased level of MDA and decreased level of SOD activity and GSH-Px activity in D-galactose induced aging mice (30) and D-galactose induced aging rats (31). Our data revealed similar results in D-galactose induced aging dogs, compared with young control and naturally aging dogs, suggesting that aging dogs have more oxidative stress and free radical but less anti-oxidant and enzymes to handle these stress resulting in serious damage in tissue and organs. Furthermore, decreased level of PCNA and elevated level of p16 and p21 has been considered as a standard event in aging (32). Results from the present study confirmed that D-galactose induced aging dogs has a similar aging-related factors expression profile to naturally aging dogs, including reduced level of PCNA, and high level of NF- $\mathrm{B}$, iNOS, COX2, p16 and p21. These aging-related markers are driving a number of apoptosis, necrosis, tissue remodeling and inflammatory response signaling pathways that consisting of the complex senescence processes leading to aging.

To the best of our knowledge, our study showed for the first time D-galactose induced aging beagle dogs used as an aging model. These results provides a strong piece of evidence supporting that D-galactose induced aging model as a well-established model in mice and rats, can also be applied in beagle dogs. This accelerated induced aging model might be promising tool for human aging in the future. In addition, our comparison study extends the understanding of the etiology 
of aging-related progression in different organs and also the important implication in the investigations on age-related diseases.

\section{Acknowledgements}

This study was funded by Provincial Science and Technology Project of Guangdong Province (no. 2015A030302076, 2016A030303056 and 2016A020215224) and the Natural Science Foundation Project of Guangdong Province (no. 2015A030310046 and 2016A030313674).).

\section{References}

1. Dillin A, Gottschling DE and Nyström T: The good and the bad of being connected: The integrons of aging. Curr Opin Cell Biol 26: 107-112, 2014.

2. Niedernhofer LJ, Kirkland JL and Ladiges W: Molecular pathology endpoints useful for aging studies. Ageing Res Rev 35: 241-249, 2017

3. Marchal J, Pifferi F and Aujard F: Resveratrol in mammals: Effects on aging biomarkers, age-related diseases, and life span. Ann NY Acad Sci 1290: 67-73, 2013.

4. Mitchell SJ, Scheibye-Knudsen M, Longo DL and de Cabo R Animal models of aging research: Implications for human aging and age-related diseases. Annu Rev Anim Biosci 3: 283-303, 2015.

5. Lees $\mathrm{H}$, Walters $\mathrm{H}$ and Cox LS: Animal and human models to understand ageing. Maturitas 93: 18-27, 2016.

6. Harkema L, Youssef SA and de Bruin A: Pathology of mouse models of accelerated aging. Vet Pathol 53: 366-389, 2016.

7. Liao CY and Kennedy BK: Mouse models and aging: Longevity and progeria. Curr Top Dev Biol 109: 249-285, 2014.

8. Gurkar AU and Niedernhofer LJ: Comparison of mice with accelerated aging caused by distinct mechanisms. Exp Gerontol 68: 43-50, 2015.

9. Head E: A canine model of human aging and Alzheimer's disease. Biochim Biophys Acta 1832: 1384-1389, 2013.

10. Bosch MN, Pugliese M, Gimeno-Bayón J, Rodríguez MJ and Mahy N: Dogs with cognitive dysfunction syndrome: A natural model of Alzheimer's disease. Curr Alzheimer Res 9: 298-314, 2012.

11. Araujo JA, Nobrega JN, Raymond R and Milgram NW: Aged dogs demonstrate both increased sensitivity to scopolamine impairment and decreased muscarinic receptor density. Pharmacol Biochem Behav 98: 203-209, 2011.

12. Vasilevko V and Head E: Immunotherapy in a natural model of Abeta pathogenesis: The aging beagle. CNS Neurol Disord Drug Targets 8: 98-113, 2009.

13. Shahroudi MJ, Mehri S and Hosseinzadeh H: Anti-aging effect of nigella sativa fixed oil on D-galactose-induced aging in mice. J Pharmacopuncture 20: 29-35, 2017.

14. Liu C, Hu J, Mao Z, Kang H, Liu H, Fu W, Lv Y and Zhou F: Acute kidney injury and inflammatory response of sepsis following cecal ligation and puncture in d-galactose-induced aging rats. Clin Interv Aging 12: 593-602, 2017.

15. Parameshwaran $\mathrm{K}$, Irwin $\mathrm{MH}$, Steliou $\mathrm{K}$ and Pinkert $\mathrm{CA}$ $\mathrm{D}$-galactose effectiveness in modeling aging and therapeutic antioxidant treatment in mice. Rejuvenation Res 13: 729-735, 2010 .
16. Ho SC, Liu JH and Wu RY: Establishment of the mimetic aging effect in mice caused by D-galactose. Biogerontology 4: 15-18, 2003.

17. Soria-Valles C, Osorio FG, Gutiérrez-Fernández A, De Los Angeles A, Bueno C, Menéndez P, Martín-Subero JI, Daley GQ, Freije JM and López-Otín C: NF-kB activation impairs somatic cell reprogramming in ageing. Nat Cell Biol 17: 1004-1013, 2015.

18. Harada CN, Natelson Love MC and Triebel KL: Normal cognitive aging. Clin Geriatr Med 29: 737-752, 2013.

19. Maes C, Gooijers J, Orban de Xivry JJ, Swinnen SP and Boisgontier MP: Two hands, one brain, and aging. Neurosci Biobehav Rev 75: 234-256, 2017.

20. Fast R, Schütt T, Toft N, Møller A and Berendt M: An observational study with long-term follow-up of canine cognitive dysfunction: Clinical characteristics, survival, and risk factors. J Vet Intern Med 27: 822-829, 2013.

21. Freeman LM: Cachexia and sarcopenia: Emerging syndromes of importance in dogs and cats. J Vet Intern Med 26: 3-17, 2012.

22. Kim SA, Lee KH, Won HY, Park S, Chung JH, Jang Y and Ha JW: Quantitative assessment of aortic elasticity with aging using velocity-vector imaging and its histologic correlation. Arterioscler Thromb Vasc Biol 33: 1306-1312, 2013.

23. Kraus C, Pavard S and Promislow DE: The size-life span trade-off decomposed: Why large dogs die young. Am Nat 181: 492-505, 2013.

24. Jucker M: The benefits and limitations of animal models for translational research in neurodegenerative diseases. Nat Med 16 1210-1214, 2010.

25. Borras D, Ferrer I and Pumarola M: Age-related changes in the brain of the dog. Vet Pathol 36: 202-211, 1999.

26. Schriefer JA and Spratto GR: Examination of dihydrotachysterolinduced progeria as a model for aging changes in carbohydrate metabolism. J Pharmacol Methods 3: 297-304, 1980.

27. Xing H, Hu X, Liu H, Li Y and Chen Y: Study on DNA oxidative damage of $\mathrm{O} 3$ aging model in mice. Hua Xi Yi Ke Da Xue Xue Bao 32: 229-231, 2001 (In Chinese).

28. Budni J, Garcez ML, Mina F, Bellettini-Santos T, da Silva S, Luz APD, Schiavo GL, Batista-Silva H, Scaini G, Streck EL and Quevedo J: The oral administration of D-galactose induces abnormalities within the mitochondrial respiratory chain in the brain of rats. Metab Brain Dis 32: 811-817, 2017.

29. Murata M, Ohta N, Fujisawa S, Tsai JY, Sato S, Akagi Y, Takahashi Y, Neuenschwander $\mathrm{H}$ and Kador PF: Selective pericyte degeneration in the retinal capillaries of galactose-fed dogs results from apoptosis linked to aldose reductase-catalyzed galactitol accumulation. J Diabetes Complications 16: 363-370, 2002.

30. Calvert GD and Scott PJ: Properties of two pig low density lipoproteins prepared by zonal ultracentrifugation. Atherosclerosis 22: 583-599, 1975.

31. Li M, Ouyang W, Wu X, Zheng Y, Wei Y and An L: Kinetin inhibits apoptosis of aging spleen cells induced by D-galactose in rats. J Vet Sci 15: 353-359, 2014

32. Stein GH, Drullinger LF, Soulard A and Dulić V: Differential roles for cyclin-dependent kinase inhibitors p21 and p16 in the mechanisms of senescence and differentiation in human fibroblasts. Mol Cell Biol 19: 2109-2117, 1999. 